\title{
A large soluble domain of the Staphylococcus aureus ESAT-6 Virulence Factor EsaA is stable in the absence of its cognate transmembrane domains
}

\author{
Manar M. Ahmed ${ }^{\mathrm{a}}$, and Yasser M. Ragab ${ }^{\mathrm{b}}$, Khaled M. Aboshanab $^{\mathrm{c}^{*}}$ \\ ${ }^{a}$ Department of Microbiology and Immunology, Faculty of Pharmacy and Pharmaceutical Industries, Sinai \\ University, El-Arish, North Sinai, Egypt \\ ${ }^{b}$ Department of Microbiology and Immunology, Faculty of Pharmacy, Cairo University, Cairo, Egypt \\ ${ }^{c}$ Department of Microbiology and Immunology, Faculty of Pharmacy, Ain Shams University, Cairo, Egypt
}

\begin{abstract}
Staphylococcus (s.) aureus is a commensal microorganism with a significant threat to human health. S. aureus harbors secretion systems that are utilized to exploit host cells through the secretion of a subset of virulence proteins that cause serious illness. The ESAT6-like or Type VII Secretion System (T7SS) contributes to S. aureus virulence. The T7SS secretion system encodes at least twelve proteins categorized as cytosolic, membrane-associated or secreted. Little is known about the exact components of the translocation machinery or translocation mechanism of T7SS substrates across the staphylococcal envelope, but translocation of T7SS substrates across bacterial membranes requires four membrane proteins: EsaA and EssA, B, and C. Topology predictions of EsaA suggest six transmembrane domains with large soluble stretch, likely exposed into trans side of the membrane. Whether the large soluble stretch of EsaA is stable without the need for other transmembrane domains, a recombinant 6x-Histagged soluble domain of EsaA was overproduced in Escherichia (E.) coli BL21(DE3) cells. This was followed by affinity purification of the tagged EsaA soluble over a Fast Protein Liquid Chromatography-operated nickel column to apparent homogeneity. SDS-PAGE of the affinity-purified soluble stretch without its cognate transmembrane domains revealed a strong signal, suggesting an independent role for that domain in mediating protein-protein interactions within the ESAT-6 secretion system.
\end{abstract}

Keywords: $S$. aureus; ESAT-6; Type VII secretion; EsaA-SD

*Correspondence | Khaled M. Aboshanab; Professor of Microbiology and Immunology, Faculty of Pharmacy, Ain Shams University, African union organization street Abassia, 11566, Cairo, Egypt. Email: aboshanab2012@pharma.asu.edu.eg

Citation | Manar M. Ahmed, and Yasser M. Ragab, and Khaled M. Aboshanab. 2017. A large soluble domain of the Staphylococcus aureus ESAT-6 Virulence Factor EsaA is stable in the absence of its cognate transmembrane domains. Arch Pharm Sci ASU 1(2): 39-45

DOI: $10.21608 /$ aps.2017.11025

Online ISSN: 2356-8380. Print ISSN: 2356-8399.

Received 26 July 2017. Accepted 11 October 2017.

Copyright: ${ }^{\circledR} 2017$ Ahmed et al. This is an open-access article licensed under a Creative Commons Attribution 4.0 International License (CC BY 4.0), which permits unrestricted use, distribution, and reproduction in any medium, provided the original author(s) and source are credited. Published by: Ain Shams University, Faculty of Pharmacy.

\section{INTRODUCTION}

$S$. aureus is a commensal bacterium with a significant threat to public health $[\mathbf{1}, 2]$. Under some cases of skin injury, innate immune barriers are breached and S. aureus gains access into deeper tissues, leading to persistent staphylococcal abscesses in host tissues without promoting immune response [3]. S. aureus develops secretion systems to export their virulence factors extracellularly and into the vicinity of their host environment [4].

ESAT-6 (early secreted antigenic targets of 6 $\mathrm{kDa}$ ) or Type VII Secretion System (T7SS) is a specialized secretion system that contributes to $S$. aureus virulence [5]. It is evolutionarily related to the ESX-1 secretion system, initially identified 
in Mycobacterium tuberculosis during the isolation of the BCG vaccine strain Mycobacterium bovis [6]. Genomic data analysis show, that ESAT-6 is conserved in other Grampositive Firmicutes such as most of $S$. aureus strains [7], Bacillus subtilis and S. agalactiae [8]. The $S$. aureus T7SS encodes at least twelve proteins categorized as cytosolic (EsaB), four membrane-associated proteins (EsaA, EssA, EssB, and EssC), and several secreted proteins (EsxA, EsxB, EsxC, EsxD, and EssD). The two remaining genes encode, EsaE; a membrane platform protein and EssaG, an EssD nuclease [4, 9, 10]. The EsxA and EsxB WXG100 secreted proteins are essential for persistence of staphylococcal abscesses as indicated by murine abscess model experiments [4]. Furthermore, EsxC and EsxD are also secreted and essential for staphylococcal virulence. The hallmark of $S$. aureus T7SS is the secretion of EsxABCD that strongly manipulate host defense mechanisms [4, $8,10]$.

Little is known about the translocation machinery or translocation mechanism of T7SS substrates across the staphylococcal envelope, but, translocation of T7SS substrates across biological membranes requires four membrane proteins: EsaA, EssA, EssB and EssC [4, 5, 7]. The molecular mechanism of EssC has been assessed as FtsK/SpoIIIE homolog with ATPase activity; probably acting to energize substrate translocation across staphylococcal envelope [11, 12]. The crystal structure of EssB shows bitopic transmembrane domain residing within the membrane. The C-terminus is likely located into trans side of the membrane and is essential for EssB homodimerization, whereas the N-terminus is located in the cytoplasm and its folding pattern resembles that of protein kinases likely recognizing several T7SS substrates [13].

The molecular mechanisms of EssA and EsaA are under ongoing investigation. Topology predictions of EsaA using TMHMM reveal six transmembrane domains embedded within the membrane and spread across protein length. In addition, EsaA contains one large soluble stretch located between TM1 and TM2 and is likely exposed into the trans side of the membrane. We wondered whether the sole large soluble stretch of EsaA membrane protein is stable without the presence of other TM domains, which suggests an independent role in mediating protein-protein interactions. In order to test this hypothesis, a systematic experimental approach has been carried out in this work to confirm this hypothesis.

\section{MATERIALS AND METHODS}

\subsection{Bacterial cultures}

E. coli cultures of DH5 $\alpha$ and BL21 (DE3) were grown in Luria Bertani (LB) medium (10 g $\mathrm{NaCl}, 10 \mathrm{~g}$ peptone, $5 \mathrm{~g}$ yeast extract) at $37{ }^{\circ} \mathrm{C}$. Ampicillin antibiotic was used at final concentration $100 \mu \mathrm{g} / \mathrm{mL}$ for plasmid selection in E. coli. Isopropyl $\beta$-D-1-thiogalactopyranoside (IPTG) with final concentration $0.5 \mathrm{mM}$ was added for gene induction, if necessary. Phenylmethylsulfonylfluoride (PMSF) as protease inhibitor at final concentration $0.5 \mathrm{mM}$ and lysozyme at $1 \mathrm{mg} / \mathrm{ml}$ were also added prior to protein purification.

\subsection{Bacterial strains and plasmid}

E. coli $\mathrm{DH} 5 \alpha$ cells were used for cloning experiments. E. coli BL21 (DE3) cells were also used for protein overproductionexperiments. $6 \mathrm{x}$ His-tagged esaA soluble part (EsaA-SD) was cloned into an expression vector pET15 busing the following oligonucleotide sequences (F: 5 CGCAGCATATGACTTTAATTGAAAAACA AAATTCATTAT 3') and (R: 5 CGCAGGGATCCTTATAAAATCACCATTAA GATGAATTTC 3').

\subsection{Cloning of esaA soluble stretch}

esaA soluble stretch was amplified by polymerase chain reaction (PCR) using genomic DNA extracted from $S$. aureus strain USA300 using Qiaminiprep as a PCR template and the aforementioned set of oligonucleotides. Next, PCR products were purified from agarose gels using standard methods and subjected to restriction endonucleases digestion using $N d e \mathrm{I}$ and BamHI. The cleaved products were then ligated into the pET15b expression vector digested with the same restriction endonucleases. Recombinant plasmid (pET15b6x esaA-SD) was 
then introduced into $E$. coli BL21 (DE3) competent cells for protein overproduction and subsequent affinity purification

\subsection{Recombinant protein affinity purification}

Plasmid pET15b6x esaA-SD was introduced into E. coli BL21 (DE3) cells. E. coli BL21 (DE3) cultures harboring pET15b6x esaA-SD were grown overnight at $37{ }^{\circ} \mathrm{C}$ in liquid $\mathrm{LB}$, supplemented with ampicillin for vector propagation. Next day, cultures were subcultured into fresh LB medium containing ampicillin and further incubated at $30{ }^{\circ} \mathrm{C}$ until absorbance values A600 $\mathrm{nm}$ reached 0.6. Thereafter, IPTG with final concentration $0.5 \mathrm{mM}$ was added, and cells were further incubated for three hours at 30 ${ }^{\circ} \mathrm{C}$. Cells were harvested by centrifugation $(12,000 \mathrm{rpm})$ while discarding the supernatant. The obtained cell pellet suspended in PBS (phosphate buffer saline) containing $20 \mathrm{mM}$ imidazole, $0.5 \mathrm{mM}$ PMSF and $1 \mathrm{mg} / \mathrm{ml}$ lysozyme and further subjected to sonication $30 \mathrm{amp}$ (5 min), $10 \mathrm{sec}$ pulse rate and $10 \mathrm{sec}$ break inbetween, followed by $30 \mathrm{amp}(6 \mathrm{~min}), 10 \mathrm{sec}$ pulse rate and $10 \mathrm{sec}$ break in-between. Crude lysates were centrifuged in a cooling centrifuge at $4000 \mathrm{rpm}$ for $20 \mathrm{~min}$ with the recovery of the filtered supernatant of extracts, producing histagged esaA-SD along with other native E. coli proteins (total cell lysates). For affinity purification, the supernatant was injected using a needle syringe into immobilized metal affinity chromatography (IMAC) over an FPLC-attached His-Trap nickel column (GE Healthcare). The column was washed with phosphate buffer saline, pH 7.5 containing $20 \mathrm{mM}$ imidazole as wash buffer, followed by gradual elution in the presence of phosphate buffer saline (PBS) containing $500 \mathrm{mM}$ imidazole. The eluted fractions of 6x-His-tagged EsaA-SD were concentrated with acetone (3:1 and incubated on ice for $30 \mathrm{~min}$, then centrifuged for $45 \mathrm{~min}$ at $4000 \mathrm{rpm}$, room temperature. Acetone treated elution fractions were air dried. Proteins in all fractions (TCL: total cell lysates, FT: flow through, W: wash and EL: elution) were identified using sodium dodecyl sulfate poly Acrylamide gel electrophoresis (SDS-PAGE).

\subsection{SDS-PAGE protocol}

Following protein affinity purification, standard sodium dodecyl sulfate-polyacrylamide gel electrophoresis (SDS-PAGE) was performed to validate EsaA-SD production and stability. Protein fractions (BL21EV, TCL, FT, W and EL) were mixed with sample buffer and incubated for 5 min at $95{ }^{\circ} \mathrm{C}$. Samples were loaded onto an SDS-PAGE gel [14], followed by coomassie blue staining $(300 \mathrm{~mL}$ methanol, $100 \mathrm{~mL}$ glacial acetic acid, $600 \mathrm{~mL}$ distilled water and $1 \mathrm{~g}$ Coomassie Blue R250) to detect the protein signal at $\sim 70 \mathrm{kDa}$. De-staining solution $(200 \mathrm{~mL}$ methanol, $80 \mathrm{~mL}$ of glacial acetic acid, and 720 $\mathrm{mL}$ distilled water) was used to remove excess staining and manifest band detection.

\subsection{Membrane topology predictions}

Tied Mixture Hidden Markov Model (TMHMM) server (http://www.cbs.dtu.dk/services/TMHMM) was used as software for topology predictions of the EsaA membrane protein. EsaA amino acid sequence was introduced into the server in a FASTA format, followed by detection of the protein topology as outlined by the web-based tool.

\section{RESULTS AND DISCUSSION}

\subsection{The EsaA soluble stretch is stable without its cognate transmembranes}

As shown in Fig. 1, topology prediction of EsaA showed that it is an integral membrane protein with 1009 amino acids comprising six transmembrane domains spread throughout the protein length, with large soluble stretch likely exposed into the trans side of the membrane. EsaA protein sequence: $\mathrm{N}$ - terminus (1-4 amino acids), TM1 (5-27 aa), soluble stretch (28-820 aa), TM2 (821-843 aa), TM3 (864-886 aa), TM4 (901-923 aa), TM5 (928-945 aa), TM6 (9781000 aa). To confirm whether the sole large soluble stretch of EsaA is stable in the absence of cognate TM, an affinity purified6x-His-tagged EsaA soluble stretch (EsaA His-SD) was performed upon overproduction in E. coli BL21 (DE3) cells using IMAC, over an FPLC-operated His-Trap nickel column (GE Healthcare). Different samples (TCL for total cell lysates, FT 
for flow through, WS for wash and EL for elution) were collected based on the chromatogram chart (Fig. 2).

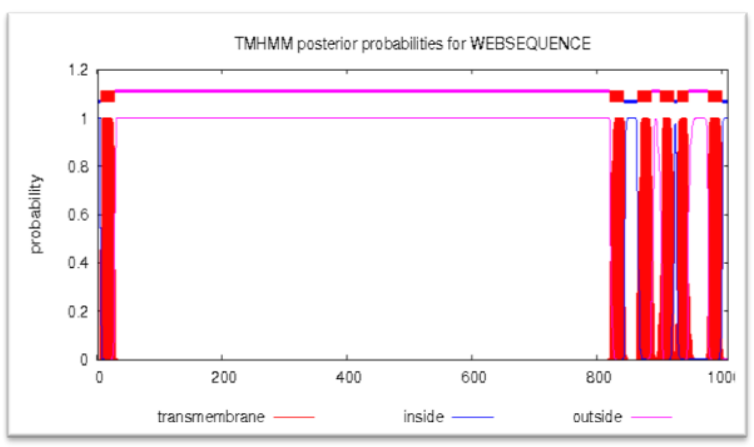

Fig. 1 TMHMM Topology prediction of EsaA integral membrane protein

EsaA is integral membrane protein with1009 amino acids comprising six transmembrane domains spread throughout the protein length, with large soluble stretch likely exposed into the trans side of the membrane. EsaA protein sequence: $\mathrm{N}$ - terminus (1-4 amino acids), TM1 (5-27 aa), soluble stretch (28-820 aa), TM2 (821843 aa), TM3 (864-886 aa), TM4 (901-923 aа), TM5 (928-945 aa), TM6 (978-1000 aa).

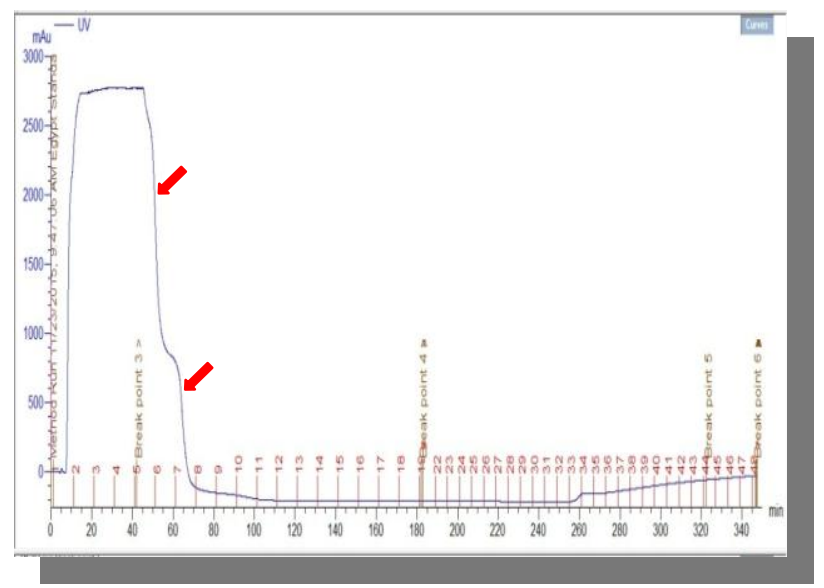

Fig. 2 FPLC Chromatogram chart of the EsaA-SD purification, Arrows are flowed through and wash.

Chromatogramrepresentsflow through, wash and elution fractions after injecting the supernatant sample (containing 6x-His-tagged EsaA-SD) into IMAC over an FPLC-attached His-Trap nickel column (GE Healthcare). Flow through the sample (fraction 5-6), Wash sample (fractions 7-15) and elution samples (36-40).

\subsection{SDS-PAGE analysis of EsaA}

An additional band of SD-EsaA of approximately $\sim 70 \mathrm{kDa}$ appeared in the total cell lysate (TCL) (Fig. 3, lane 3 left) that refers to successful expression of the SD-EsaA, while it does not show in flow-through sample (FT), as the flow-through sample includes all proteins except His-tagged protein (His-tagged EsaASD), as shown in (Fig. 3 lane 4 left). The washed sample indicates the successful removal of non 6x-His-tagged proteins traces. EsaA-SD elution fractions (36-40) in the chromatogram (Fig. 2) was concentrated using acetone followed by SDS-PAGE, in elution fraction, and a protein band appeared in approximately $\sim 70 \mathrm{kDa}$, where EsaA-SD is supposed to appear near this molecular size (Fig. 4, lane 5).

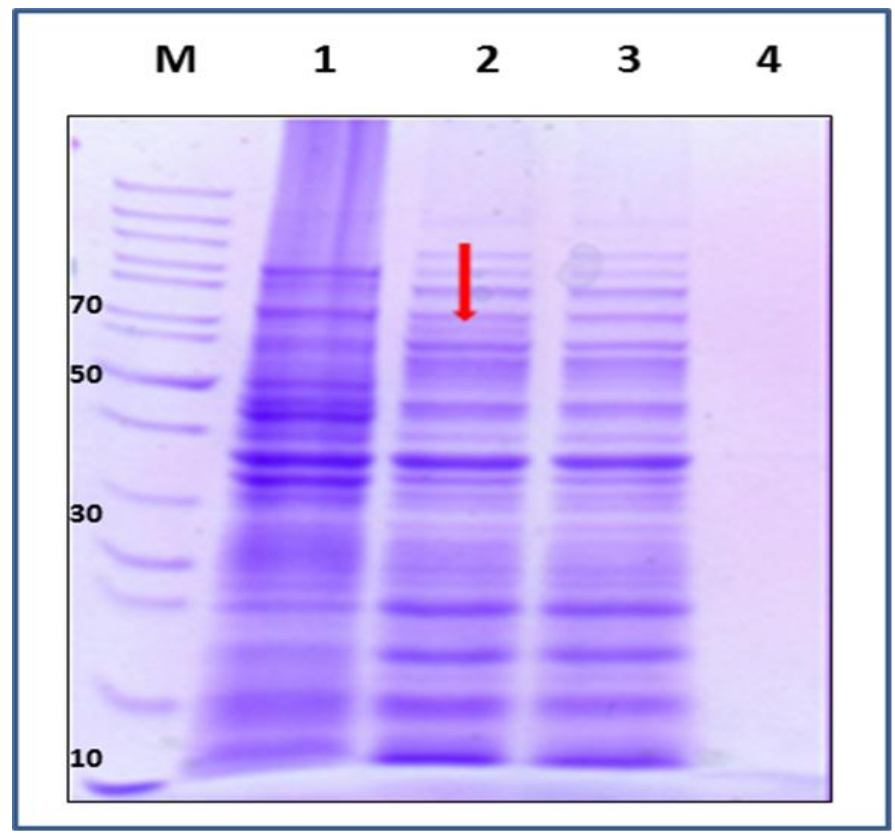

Fig. 3 Coomassie-stained SDS-PAGE of FPLC fractions of affinity purified EsaA-SD.

SDS-PAGE was performed for analysis of total cell lysates containing E. coli proteins, including 6x-His-tagged EsaA-SD, Flow through sample and wash sample using E. coli BL21 EV as a control. Lanes from left to right: M; Protein 
molecular weight marker (10-200 kDa), lane 1; BL21-EV cells following induction with IPTG as a negative control, lane 3; (TCL) crude lysates of BL21-pET15 harboring EsaA (SD), lane 4; (FT) Flow-through,5; Wash.

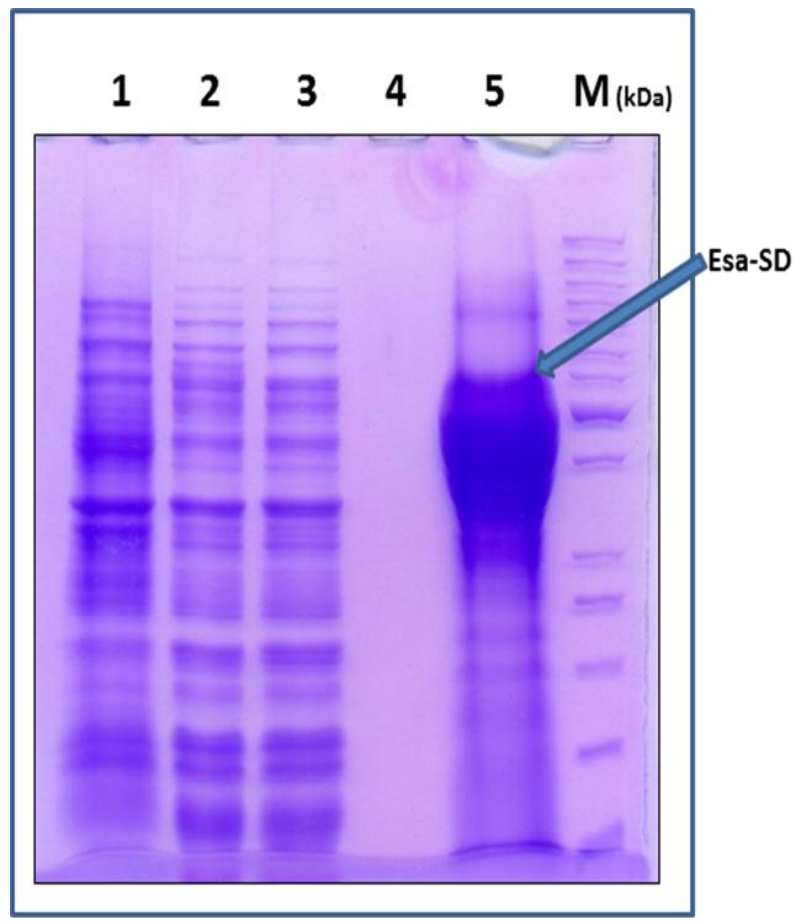

Fig. 4 SDS-PAGE of FPLC concentrated EsaA-SD elution fractions

EsaA-SDelution fractions (36-40) are subjected to concentration with three times absolute acetone solution. SDS-PAGE represents concentrated elution sample with total cell lysates, flow through and wash using BL21-EV as a negative control. Lanes from left to right: Lane 1; BL21-EV cells following induction with IPTG as negative control, Lane 2; crude lysates of BL21-pET15b harboring EsaA (SD), Lane 3; flow-through sample representing all proteins except 6x-His-tagged EsaA-SD, Lane 4; wash, lane 5; concentrated EsaA-SD elution fractions $\sim 70 \mathrm{kDa}$, lane $\mathrm{M}$;Protein molecular weight marker (10-200 kDa).

\section{DISCUSSION}

The ESAT-6 secretion pathway is crucial for pathogenicity of many human pathogens, such as $S$. aureus and $M$. tuberculosis. Membrane proteins often show higher importance in many physiological mechanisms including structural and functional integrity and it is not a coincidence that they are targeted by over $50 \%$ of marketed drugs [15]. The S. aureus ESAT-6 system is composed of four integral membrane proteins: EsaA, EssBA and EssC, an associated ATPase, which are all required for protein secretion across the staphylococcal envelope [4, 5, 16]. EsaA exhibits six transmembrane domains with a large extracellular loop.

In this study, we have investigated whether the sole large soluble stretch of EsaA is stable without the need for other transmembrane domains using affinity purification. Our results using affinity purification of a 6x-His-tagged EsaA soluble stretch performed over a Fast Protein Liquid Chromatography-operated nickel column reveal the stability of EsaA soluble stretch without its cognate transmembrane domains, suggesting an independent role in protein-protein interactions within the ESAT-6 secretion system.

Previous studies on membrane proteins harboring long extracellular loops develop many roles. For example, the $S$. aureus BraSR/BraDE/VraDE (for Bacitracin resistance associated) system, and the extracellular loop of VraE has been shown to associate bacitracin resistance [17]. Further, the BceB extracellular loop in B. subtilis is thought to be necessary for bacitracin sensing and resistance $[\mathbf{1 8}, \mathbf{1 9}]$.

Similar to the EsaA extracytoplasmic loop, studies have shown that conserved extracytoplasmic loops in other bacteria including B. subtilis and E. coli may exhibit important functional mechanisms. Interestingly, short deletions in the E. coli Sec Dextra cytoplasmic loop result in malfunctioning of the protein without affecting the stability of SecDF complexes with others, suggesting its participation in providing a protective structure for efficient protein folding [1]. Moreover, in $B$. subtilis, topology predictions of SecDF also show besides its 12 transmembranes, two extracytoplasmic loops conserved between TM1TM2 and TM7-TM8, where studies show SecD and $\mathrm{SecF}$ with deletion in the large cytoplasmic 
loop, displayed translocation defect without affecting SecD-SecF interaction,reported that it is important for catalyzing protein translocation $[1,20]$. The exact function of extracytoplasmic loops is not completely understood, but they may participate as binding sites for lipids [21] or associate with lipids for correct insertion, folding, and proper topology of the protein [22]. In addition, studies of $\mathrm{C} 2$ domain structure show surface loops that are required for domain specificity, with their amino acid sequence and conformation varying and mostly involved with lipid binding [23]. Future studies will be performed in order to understand the exact function of the EsaA extracytoplasmic loop and its role with other membrane proteins within the ESAT-6 pathway.

\section{CONCLUSION}

SDS-PAGE analysis of the expressed soluble stretch of the EsaA membrane protein, a major protein of the ESAT-6 secretion system of Staphylococcus aureus that contributes to its virulence showed its stability without its cognate transmembrane domains revealed strong signal, suggesting an independent role for that domain in mediating protein-protein interactions within the ESAT-6 secretion system or Ttype VII Secretion System (T7SS)

\section{Acknowledgment}

We acknowledge the Department of Microbiology and Immunology, Faculty of Pharmacy and Pharmaceutical Industries, Sinai University, El-Arish, North Sinai, Egypt for providing all required Laboratory facilities when required as well as their friendly cooperation.

\section{Conflict of Interest}

The author declares no Conflict of Interest

\section{REFERENCES}

1. Sibbald MJ, Ziebandt AK, et al. Mapping the pathways to staphylococcal pathogenesis by comparative secretomics. Microbiol Mol Biol Rev 2006; 70(3): 755-788.

2. Bartlett AH, Hulten KG. Staphylococcus aureus pathogenesis: secretion systems, adhesins, and invasions. Pediatr Infect Dis J 2010; 29(9): 860861 .

3. Anderson M, Chen $\mathrm{YH}$ et al. EsaD, a secretion factor for the Ess pathway in Staphylococcus aureus. J Bacteriol 2011; 193(7): 1583-1589.

4. Burts ML, W. A. Williams, et al. EsxA and EsxB are secreted by an ESAT-6-like system that is required for the pathogenesis of Staphylococcus aureus infections. Proc Natl Acad Sci USA 2005; 102(4): 1169-1174.

5. Kneuper H, Cao ZP, et al. Heterogeneity in ess transcriptional organization and variable contribution of the Ess/Type VII protein secretion system to virulence across closely related Staphylococcus aureus strains. Mol Microbiol 2014; 93(5): 928-943.

6. Abdallah AM, Gey van Pittius NC, et al. Type VII secretion--mycobacteria show the way. Nat Rev Microbiol 2007; 5(11): 883-891.

7. Warne B, Harkins CP, et al. The Ess/Type VII secretion system of Staphylococcus aureus shows unexpected genetic diversity. BMC Genomics 2016; 17: 222.

8. Burts ML, DeDent AC, et al. EsaC substrate for the ESAT-6 secretion pathway and its role in persistent infections of Staphylococcus aureus. Mol Microbiol 2008; 69(3): 736-746.

9. Cao Z, Casabona MG, et al. The type VII secretion system of Staphylococcus aureus secretes a nuclease toxin that targets competitor bacteria. Nat Microbiol 2016; 2: 16183.

10. Anderson M, Aly KA, et al. Secretion of atypical protein substrates by the ESAT-6 secretion system of Staphylococcus aureus. Mol Microbiol 2013; 90(4): 734-743.

11. Tanaka Y, Kuroda M, et al. Crystal structure analysis reveals a novel forkhead-associated domain of ESAT-6 secretion system C protein in Staphylococcus aureus. Proteins 2007; 69(3): 659-664.

12. Zoltner $\mathrm{M}$, $\mathrm{Ng} \mathrm{WM}$, et al. EssC: domain structures inform on the elusive translocation channel in the Type VII secretion system. Biochem J 2016; 473(13): 1941-1952.

13. Zoltner M, Norman DG, et al. The architecture of EssB, an integral membrane component of the type VII secretion system. Structure 2013; 21(4): 
595-603.

14. Schagger H . Coomassie blue-sodium dodecyl sulfate-polyacrylamide gel electrophoresis for direct visualization of polypeptides during electrophoresis. Anal Biochem.1988

15. Souda P, Ryan CM, et al. Profiling of integral membrane proteins and their post-translational modifications using high-resolution mass spectrometry. Methods2011; 55(4): 330-336.

16. Jager F, Zoltner M, et al. Membrane interactions and self-association of components of the Ess/Type VII secretion system of Staphylococcus aureus. FEBS Lett 2016; 590(3): 349-357.

17. Hiron A, Falord M, et al.Bacitracin and nisin resistance in Staphylococcus aureus: a novel pathway involving the BraS/BraR twocomponent system (SA2417/SA2418) and both the $\mathrm{BraD} / \mathrm{BraE}$ and $\mathrm{VraD} / \mathrm{VraE} \mathrm{ABC}$ transporters. Mol Microbiol 2011; 81(3): 602-622.

18. Rietkotter E, Hoyer D, et al. Bacitracin sensing in Bacillus subtilis. Mol Microbiol 2008; 68(3): 768-785.

19. Coumes-Florens S, Brochier-Armanet C, et alA new highly conserved antibiotic sensing/resistance pathway in firmicutes involves an $\mathrm{ABC}$ transporter interplaying with a signal transduction system. PLoS One 2011; 6(1): e15951.

20. Nouwen N, Piwowarek M, et al. The large first periplasmic loop of $\mathrm{SecD}$ and $\mathrm{SecF}$ plays an important role in SecDF functioning. J Bacteriol 2005; 187(16): 5857-5860.

21. Laganowsky A, Reading E, et al. Membrane proteins bind lipids selectively to modulate their structure and function. Nature 2014; 510(7503): 172-175.

22. Palsdottir H, Hunte C. Lipids in membrane protein structures. Biochim Biophys Acta 2004; 1666(12): 2-18.

23. Stahelin RV Lipid binding domains: more than simple lipid effectors. J Lipid Res 2009; 50 Suppl: S299-304.2 